\title{
Prevalence, molecular characterization, and antimicrobial resistance profiles of Listeria monocytogenes, Salmonella enterica, and Escherichia coli 0157:H7 on dairy cattle farms in Jordan
}

\author{
Mohammad M. Obaidat ${ }^{1 *}$ and Andrew P. Stringer ${ }^{2}$ \\ ${ }^{1}$ Department of Veterinary Pathology and Public Health, Faculty of Veterinary Medicine, Jordan University of Science and Technology, Irbid 22110, \\ Jordan \\ ${ }^{2}$ Department of Population Health and Pathobiology, College of Veterinary Medicine, North Carolina State University, Raleigh 27607
}

\section{ABSTRACT}

This study determined the prevalence, pulsed-field gel electrophoresis profiles, and antimicrobial resistance profile of Listeria monocytogenes, Salmonella enterica, and Escherichia coli O157:H7 isolates from dairy cattle farms in Jordan. Samples from bulk tank milk $(\mathrm{n}=305)$, cattle feces $(\mathrm{n}=610)$, and rectoanal mucosal swabs $(\mathrm{n}=610)$ were collected from 61 dairy cattle farms. We confirmed 32 L. monocytogenes, 28 S. enterica, and 24 E. coli $\mathrm{O} 157: \mathrm{H} 7$ isolates from the samples. The farm-level prevalence (at least 1 positive sample per farm) of L. monocytogenes, S. enterica, and E. coli $\mathrm{O} 157$ :H7 was $27.9,19.7$, and $23.0 \%$, respectively. The prevalence of $L$. monocytogenes, $S$. enterica, and $E$. coli O157:H7 in bulk tank milk was 7.5, 1.6, and 3.3\%, respectively. The prevalence of $L$. monocytogenes and $S$. enterica in fecal samples was 1.5 and $3.8 \%$, respectively, and the prevalence of E. coli $\mathrm{O} 157: \mathrm{H} 7$ in rectoanal mucosal swabs was $2.3 \%$. Based on disk diffusion testing, all L. monocytogenes, S. enterica, and E. coli O157:H7 isolates exhibited resistance to at least 1 antimicrobial class. Multidrug resistance (resistance to 3 or more classes of antimicrobials) was exhibited by $96.9 \%$ of $L$. monocytogenes, $91.7 \%$ of E. coli $\mathrm{O} 157: \mathrm{H} 7$, and $82.1 \%$ of S. enterica isolates. Moreover, 93.8, 79.2, and $57.1 \%$ of the L. monocytogenes, E. coli O157:H7, and S. enterica isolates, respectively, were resistant to 5 or more antimicrobial classes. More than $50 \%$ of L. monocytogenes isolates were resistant to ampicillin, clindamycin, penicillin, erythromycin, quinupristin-dalfopristin, streptomycin, teicoplanin, linezolid, vancomycin, kanamycin, and tetracycline. More than $50 \%$ of $S$. enterica and $E$. coli O157:H7 isolates were resistant to ampicillin, cephalothin, nalidixic acid, kanamycin, streptomycin, amoxicillin-clavulanic acid, and tetracycline. The prevalence

Received February 18, 2019.

Accepted May 28, 2019.

*Corresponding author: mmobaidat@just.edu.jo of the studied pathogens this study was comparable to reports from other countries. The isolated pathogens exhibited a high degree of antimicrobial resistance, suggesting that the bacterial flora of dairy cattle in Jordan are under intense antimicrobial selection pressure. Additional research is required to determine the causes and drivers of resistance, and to develop approaches to mitigating antimicrobial resistance.

Key words: foodborne, pathogens, antibiotic, farm animals

\section{INTRODUCTION}

Farm management practices are one of the crucial control points for the production of a safe milk supply. However, contamination with fecal material can be challenging to prevent and can lead to milk contamination with foodborne pathogens such as Listeria monocytogenes, Salmonella enterica, and Escherichia coli O157:H7. Despite the effects of milk pasteurization and other heat treatment methods to ensure the safety of milk and milk products, minimizing the bacterial load of foodborne pathogens in unpasteurized milk is important. In addition, the consumption of raw milk and dairy products continues to grow despite the high-risk potential of these products in transmitting foodborne pathogens, as reported in the United States (Mungai et al., 2015). In Jordan, a substantial percentage of participants in one seroprevalence study reported consuming raw milk and dairy products, and this was significantly associated with Campylobacter jejuni seropositivity (Obaidat, 2019). In addition, some cheese products in the Middle East, including Jordan, are made from unpasteurized milk (Hilali et al., 2011).

Listeria monocytogenes is a major cause of foodborne infections, with a $20 \%$ case fatality rate (Scallan et al., 2011). Milk and dairy products have been associated with several human listeriosis outbreaks (CDC, 2008; Jackson et al., 2011; Langer et al., 2012; Pouillot et al., 2016). In addition, L. monocytogenes has been iso- 
lated from ruminants' feces (Mohammed et al., 2009; Schoder et al., 2011), feed, silage, milk, and milk products (Mohammed et al., 2009; Van Kessel et al., 2011; Giacometti et al., 2012; Sonnier et al., 2018), making it ubiquitous on dairy farms with the ability to form biofilms in milk harvesting equipment (Latorre et al., 2010). Both clinically infected and healthy animals excrete L. monocytogenes in their feces, and this can contaminate the bulk tank milk (BTM). In adult humans, L. monocytogenes can cause uncomplicated febrile gastroenteritis, but maternal-fetal/neonatal listeriosis in pregnant women can involve septicemia with or without cerebral infections such as meningoencephalitis, meningitis, rhombencephalitis, or brain abscesses. As well, L. monocytogenes can cause these severe symptoms in people who are elderly or immunocompromised (Fernández Guerrero et al., 2012; Jensen et al., 2016).

Salmonella enterica ssp. enterica is a common zoonotic pathogen and a leading cause of foodborne bacterial infections worldwide (WHO, 2015). Healthy dairy cattle are a potential reservoir of $S$. enterica. Similar and closely related genotypes of $S$. enterica are circulating in human and animal populations (Eguale et al., 2018; Pornsukarom et al., 2018). Molecular typing has identified matching strains among isolates from dairy cattle and humans (Rodriguez-Rivera et al., 2014). Multidrug-resistant (resistant to 3 or more classes of antimicrobials) $S$. enterica is a worldwide concern because of the limited effectiveness of first-line antimicrobials, especially for pediatric infections, and because drug-resistant $S$. enterica tends to cause prolonged or severe infections (WHO, 2017). The presence of multidrug-resistant $S$. enterica strains in ruminants, and consequently in meat and dairy products, may cause drug-resistant infections in humans (Adhikari et al., 2009; Langer et al., 2012; Van Kessel et al., 2013; Afema et al., 2015).

Escherichia coli O157:H7 is a major foodborne pathogen that colonizes the distal colon of cattle (Arthur et al., 2010). Although cattle serve as asymptomatic shedders of E. coli $\mathrm{O} 157: \mathrm{H} 7$, this pathogen causes symptoms in humans such as bloody diarrhea, severe stomach cramps, hemolytic uremic syndrome, and death (Pennington, 2010). Dairy products have been associated with $E$. coli O157:H7 outbreaks (Langer et al., 2012; Heiman et al., 2015; Currie et al., 2018). Recent findings indicated that $E$. coli $\mathrm{O} 157: \mathrm{H} 7$ infections from dairy products are more severe, have higher rates of hospitalization and hemolytic uremic syndrome, and occur more frequently in children compared to infections associated with beef consumption (Heiman et al., 2015).

The use of antimicrobials in livestock agriculture is constantly being re-evaluated, with humans and animals linked through environmental reservoirs (Holmes et al., 2016; Helke et al., 2017; Pornsukarom et al., 2018). Numerous studies worldwide have explored the antimicrobial resistance and subtypes of foodborne pathogens in dairy ruminants. However, despite the importance of dairy farms as a source of milk and milk products in Jordan, no data are available on the prevalence and antimicrobial resistance of foodborne pathogens in these farms. Bulk tank milk has a valuable role in detecting pathogens and monitoring the dynamics of antimicrobial resistance on dairy farms (Berge et al., 2007). This study aimed to determine the prevalence, molecular characterization, and antimicrobial resistance profiles of L. monocytogenes, S. enterica, and E. coli O157:H7 on dairy cattle farms in Jordan.

\section{MATERIALS AND METHODS}

\section{Dairy Production in Jordan}

The dairy ruminant population in Jordan has been described previously (Obaidat et al., 2018). Briefly, the dairy industry in Jordan includes cattle, sheep, and goat dairy farms. This is an important agricultural sector in the country, and contributes $\$ 160$ million per year to the national gross domestic product. Dairy cattle are managed in 2 farming systems, large and small, but both raise Holstein-Friesian cows. The large system is concentrated in the northeast (Al-Dulial area) and accounts for $60 \%$ of the milk production; it uses modern management practices with zero grazing. The small system is distributed over several regions of Jordan, but mostly located in the northern highlands. In this system, farmers raise cattle in small brick barns; they depend on grazing during the spring months and on feed rations during the rest of the year.

\section{Sampling}

The number of farms selected was based on dairy farm densities as provided by the Jordanian Department of Statistics. A total of 610 fecal samples, 610 rectal swabs, and 305 BTM samples were collected from 61 dairy farms in Al-Dulail $(\mathrm{n}=40)$ and the highlands (n $=21$ ) between March and July 2016. On each farm, 10 fecal and 10 rectoanal mucosal swab (RAMS) samples were collected randomly from clinically healthy animals by dividing the total number of animals per farm by 10 , giving a value $k$, and sampling every $k$ th animal. During the farm visits, 5 milk samples $(100 \mathrm{~mL})$ were collected aseptically from each bulk tank into sterile cups and transported daily in an ice box under cold conditions to the Food Safety and Zoonotic Diseases Laboratory, Faculty of Veterinary Medicine, Jordan 
University of Science and Technology. Fecal samples $(10 \mathrm{~g})$ were collected from the distal rectum of each cow by a clean rectal sleeve and placed in sterile containers and delivered on the same day to the laboratory in an ice box under cold conditions. A new sleeve was used for each animal to avoid cross-contamination. The milk and fecal samples were placed in separate ice boxes to avoid cross-contamination. The RAMS were obtained aseptically by inserting a sterile cotton-tipped swab 2 to $5 \mathrm{~cm}$ into the anus of each animal. The swabs were rubbed approximately 3 to 4 times along the rectoanal mucosal surface (Rice et al., 2003; Greenquist et al., 2005; Davis et al., 2006). The RAMS were then mixed vigorously in $10 \mathrm{~mL}$ of ice-cold sterile tryptic soy broth (TSB) and kept on ice until shipment to the laboratory (Sheng et al., 2013; Sheng et al., 2015).

\section{Microbiological Methods}

Listeria monocytogenes was isolated by mixing 20 g (mL) of collected samples with $20 \mathrm{~mL}$ of doublestrength modified University of Vermont broth (Oxoid, Basingstoke, UK) for primary enrichment at $30^{\circ} \mathrm{C}$ for $24 \mathrm{~h}$. Then, $0.1 \mathrm{~mL}$ of the broth culture was transferred to $10 \mathrm{~mL}$ of full-strength Fraser broth (Oxoid) for secondary enrichment at $35^{\circ} \mathrm{C}$ for $24 \mathrm{~h}$. Following this, blackened Fraser broth cultures were streaked onto modified Oxford agar (Oxoid) and PALCAM agar (Oxoid) and incubated at $37^{\circ} \mathrm{C}$ for $48 \mathrm{~h}$ (examined at $24 \mathrm{~h}$ and $48 \mathrm{~h}$ ) and $37^{\circ} \mathrm{C}$ for $48 \mathrm{~h}$, respectively. Up to 3 suspected L. monocytogenes colonies (1 to $2 \mathrm{~mm}$ in diameter surrounded by a dark zone on modified Oxford agar and approximately $2 \mathrm{~mm}$ in diameter, gray-green with a black sunken center and a black halo on PALCAM agar) were transferred to tryptic soy agar (TSA; Oxoid) supplemented with $0.6 \%$ yeast extract plates and incubated for $24 \mathrm{~h}$ at $37^{\circ} \mathrm{C}$. Isolates were confirmed as $L$. monocytogenes by PCR targeting the prfa gene and using LIP1 and LIP2 primers (D'Agostino et al., 2004 ), and stored in TSB with yeast extract and $20 \%$ glycerol at $-20^{\circ} \mathrm{C}$.

Salmonella enterica were isolated as described previously (Obaidat and Bani Salman, 2017). Briefly, $20 \mathrm{~g}$ $(\mathrm{mL})$ of the sample was pre-enriched in peptone water (Oxoid) for $6 \mathrm{~h}$ under shaking at $100 \mathrm{rpm}$. The enriched broth culture $(10 \mathrm{~mL})$ was then transferred to $100 \mathrm{~mL}$ of tetrathionate broth (Oxoid) and incubated at $42^{\circ} \mathrm{C}$. The tetrathionate broth cultures were then streaked onto xylose lysine Tergitol 4 (XLT4) plates (Oxoid). Suspected S. enterica colonies (entirely black or pink to red with black centers; 3 per sample) were transferred to lysine iron agar, triple sugar iron, and urea agar slants. Isolates with typical S. enterica phenotypes on the slants were confirmed by PCR for the invA gene
(Obaidat and Bani Salman, 2017). Confirmed S. enterica isolates were stored in TSB with $20 \%$ glycerol at $-20^{\circ} \mathrm{C}$ for further characterization and antimicrobial resistance testing.

For E. coli $\mathrm{O} 157: \mathrm{H} 7$ isolation, RAMS were placed in $10 \mathrm{~mL}$ TSB (Oxoid) and then vortexed for $60 \mathrm{~s}$ before being diluted and plated onto sorbitol MacConkey agar plates (Oxoid) supplemented with cefixime $(50 \mathrm{ng} / \mathrm{mL})$ and potassium tellurite $(2.5 \mu \mathrm{g} / \mathrm{mL}$; SMAC-CT; Oxoid) and incubated at $37^{\circ} \mathrm{C}$ for $24 \mathrm{~h}$. Five suspected $E$. coli O157:H7 (sorbitol-negative colonies) on SMAC-CT were confirmed as E. coli $\mathrm{O} 157: \mathrm{H} 7$ by $\mathrm{O}$ - and $\mathrm{H}$-antigen latex agglutination (Wellcolex E. coli O157:H7, Remel; Thermo Fisher Scientific, Waltham, MA). Confirmed E. coli $\mathrm{O} 157: \mathrm{H} 7$ isolates were stored in TSB with $20 \%$ glycerol at $-20^{\circ} \mathrm{C}$.

\section{Antimicrobial Susceptibility Testing}

Listeria monocytogenes were grown on TSA with $0.6 \%$ yeast extract overnight at $37^{\circ} \mathrm{C}$ and adjusted to a 0.5 McFarland turbidity in $5 \mathrm{~mL}$ sterile normal saline tubes. Isolates were screened for susceptibility to a panel of 15 antimicrobials (Oxoid) on Mueller-Hinton agar (Oxoid) supplemented with 5\% defibrinated sheep blood (Al-Samah Company, Amman, Jordan) by the disk diffusion method (CLSI, 2014). The isolates were tested for resistance to $\beta$-lactams (ampicillin $10 \mu \mathrm{g}$, penicillin $10 \mathrm{U}$, cefotaxime $30 \mu \mathrm{g}$ ), phenicols (chloramphenicol $30 \mu \mathrm{g}$ ), lincosamides (clindamycin $10 \mu \mathrm{g}$ ), quinolones (ciprofloxacin $5 \mu \mathrm{g}$ ), macrolides (erythromycin $15 \mu \mathrm{g}$ ), aminoglycosides (gentamicin $10 \mu \mathrm{g}$, streptomycin $10 \mu \mathrm{g}$, kanamycin $50 \mu \mathrm{g}$ ), tetracyclines (tetracycline $30 \mu \mathrm{g}$ ), streptogramins [quinupristin-dalfopristin 15 $\mu \mathrm{g}$, glycopeptides (teicoplanin $30 \mu \mathrm{g}$ ), vancomycin 30 $\mu \mathrm{g}$, and oxazolidinones (linezolid $30 \mu \mathrm{g}$ ).

Salmonella enterica and E. coli $\mathrm{O} 157: \mathrm{H} 7$ were grown on TSA overnight at $37^{\circ} \mathrm{C}$ and adjusted to a $0.5 \mathrm{Mc}-$ Farland turbidity in 5-mL sterile normal saline tubes. Isolates were screened for susceptibility to a panel of 12 antimicrobials (Oxoid) on Mueller-Hinton agar (Oxoid) by the disk diffusion method (CLSI, 2014). The isolates were tested for resistance to $\beta$-lactams (ampicillin $10 \mu \mathrm{g}$, amoxicillin-clavulanic acid $30 \mu \mathrm{g}$, cephalothin $30 \mu \mathrm{g}$, and ceftriaxone $30 \mu \mathrm{g}$ ), aminoglycosides (gentamicin $10 \mu \mathrm{g}$, streptomycin $10 \mu \mathrm{g}$, kanamycin 50 $\mu \mathrm{g}$ ), quinolones (ciprofloxacin $5 \mu \mathrm{g}$, nalidixic acid 30 $\mu \mathrm{g}$ ), phenicols (chloramphenicol $30 \mu \mathrm{g}$ ), tetracyclines (tetracycline $30 \mu \mathrm{g}$ ) and folate pathway inhibitors (sulfamethoxazole-trimethoprim $25 \mu \mathrm{g}$ ).

For analysis, isolates with intermediate susceptibility to the tested antimicrobials were considered susceptible. Staphylococcus aureus ATCC 29213 and Escherichia coli ATCC 25922 were used as reference 
strains for quality control. Any isolate was considered resistant if it exhibited resistance to 1 antimicrobial or more, and considered multidrug-resistant when it exhibited resistance to 3 classes of antimicrobials or more (Magiorakos et al., 2012). Statistical analysis was performed using OpenEpi online software (Dean et al., 2015). $P$-values $<0.05$ were considered significant.

\section{Pulsed-Field Gel Electrophoresis}

The bacterial isolates were subtyped by pulsed-field gel electrophoresis (PFGE) according to the United States Centers for Disease Control and Prevention (CDC) PulseNet protocols (www.pulsenetinternational .org/protocols/pfge/). Briefly, for S. enterica and E. coli O157:H7, XbaI restriction enzyme profiles were compared using PulseNet protocols and digital images were analyzed using Bionumerics v.6.6 (Applied Maths, Austin, TX). For L. monocytogenes, AscI was used to digest genomic DNA within agarose plugs before PFGE and gel images were analyzed using Bionumerics.

\section{RESULTS}

\section{Prevalence of L. monocytogenes, S. enterica, and Escherichia coli 0157:H7}

Bulk tank milk and fecal samples were cultured for $L$. monocytogenes and S. enterica, and BTM and RAMS were cultured for E. coli O157:H7. We confirmed $32 \mathrm{~L}$. monocytogenes isolates, 9 from fecal samples and 23 from BTM samples. The BTM isolates were from 13 different farms, and the fecal isolates were from 6 different farms. Two farms were positive for both fecal and BTM samples. We also confirmed 28 S. enterica isolates, 23 from fecal samples and 5 from BTM. The BTM isolates were from 2 farms, and the fecal isolates were from 10 farms. None of the farms was positive for both fecal and BTM samples. Finally, we confirmed 24 E. coli O157:H7 isolates, 14 from RAMS and 10 from BTM. The BTM isolates were from 5 farms, and the RAMS isolates were from 9 farms. None of the farms was positive for both RAMS and BTM samples. The prevalence of $L$. monocytogenes in fecal and BTM samples was $1.48 \%$ and $7.54 \%$, respectively. The prevalence of $S$. enterica in fecal and BTM samples was $3.77 \%$ and $1.64 \%$, respectively. The prevalence of E. coli O157:H7 in RAMS and BTM samples was $2.33 \%$ and $3.28 \%$, respectively. Meanwhile, the overall farm-level prevalences (at least 1 positive sample per farm) of $L$. monocytogenes, $S$. enterica, and E. coli $\mathrm{O} 157: \mathrm{H} 7$ were $27.9 \%$ (17 positive farms, $95 \%$ CI $18.2-40.2 \%$ ), $19.7 \%$ (12 positive farms, 95\% CI $11.6-31.3 \%$ ), and $23.0 \%$ (14 positive farms, 95\% CI 14.2-34.9\%). The farm-level prevalences based on BTM analysis for L. monocytogenes, S. enterica, and E. coli $\mathrm{O} 157: \mathrm{H} 7$ were $21.3 \%$ (13 positive farms, $95 \%$ CI $12.9-33.3 \%$ ), 3.3\% (2 positive farms, 95\% CI 0.9-11.9), and $8.2 \%$ (5 positive farms, $95 \%$ CI $3.6-17.6 \%$ ). The farm-level prevalences based on fecal sample analysis for L. monocytogenes and S. enterica were $9.8 \%(6$ positive farms, $95 \%$ CI $4.6-19.8 \%$ ) and $16.4 \%$ (10 positive farms, 95\% CI 9.2-27.6\%). Finally, the farm-level prevalence based on RAMS for E. coli O157:H7 was $14.8 \%$ (9 positive farms, 95\% CI 8.0-25.7\%).

\section{Antimicrobial Resistance of L. monocytogenes, S. enterica, and E. coli 0157:H7}

All L. monocytogenes isolates exhibited resistance to at least 1 antimicrobial, and about $95 \%$ of isolates demonstrated resistance to ampicillin, clindamycin, penicillin, and erythromycin (Table 1). High resistance was also exhibited toward quinupristin-dalfopristin (88\%) and streptomycin (76\%). Between 72 and $75 \%$ of the isolates exhibited resistance to teicoplanin, linezolid, vancomycin, kanamycin, and tetracycline, and approximately 50,40 , and $30 \%$ of isolates were resistant to gentamicin, chloramphenicol, and ceftriaxone, respectively. The lowest resistance $(15.6 \%)$ was seen toward ciprofloxacin (Table 1).

All S. enterica isolates exhibited resistance to at least 1 antimicrobial (Table 2). A high percentage of the isolates exhibited resistance to ampicillin (85.7\%), cephalothin $(75.0 \%)$, nalidixic acid $(71.4 \%)$, and kanamycin (64.3\%), and approximately half of the isolates exhibited resistance to streptomycin $(55.2 \%)$, amoxicillin-clavulanic acid (57.1\%), tetracycline (53.4\%), and sulfamethoxazole-trimethoprim (50\%) (Table 2). Approximately one-third of the $S$. enterica isolates exhibited resistance to gentamicin $(35.7 \%)$, chloramphenicol $(35.7 \%)$, and ciprofloxacin $(32.0 \%)$. The lowest resistance $(17.9 \%)$ was toward ceftriaxone (Table 2 ).

All E. coli $\mathrm{O} 157: \mathrm{H} 7$ isolates exhibited resistance to at least 1 antimicrobial (Table 3). Approximately 90\% of isolates were resistant to streptomycin and cephalothin, and approximately $80 \%$ demonstrated resistance to nalidixic acid and tetracycline (Table 3). A high percentage of the isolates demonstrated resistance to amoxicillin-clavulanic acid (75.0\%), ampicillin (62.5\%), and kanamycin (58.3\%). Approximately $40 \%$ of the isolates showed resistance to sulfamethoxazole-trimethoprim (41.7\%), gentamicin (37.5\%), chloramphenicol (37.5\%), and ceftriaxone $(33.3 \%)$. The lowest resistance $(12.5 \%)$ was toward ciprofloxacin (Table 3).

Multidrug resistance (resistance to 3 or more classes of antimicrobials) was exhibited by $96.9 \%$ of L. monocytogenes, $91.7 \%$ of E. coli $\mathrm{O} 157: \mathrm{H} 7$, and $82.1 \%$ of $S$. enterica isolates (Tables 1-3). Moreover, 93.8, 79.2, and 
Table 1. Antimicrobial resistance in Listeria monocytogenes isolates from dairy cattle in Jordan, 2016

\begin{tabular}{|c|c|c|c|c|}
\hline \multirow[b]{2}{*}{ Antimicrobial (breakpoint, mm) } & \multicolumn{3}{|c|}{ Resistance, $\%$} & \multirow[b]{2}{*}{$P$-value ${ }^{1}$} \\
\hline & $\begin{array}{c}\text { Fecal isolates } \\
\quad(\mathrm{n}=9)\end{array}$ & $\begin{array}{l}\text { Milk isolates } \\
\quad(\mathrm{n}=23)\end{array}$ & $\begin{array}{c}\text { Total } \\
(\mathrm{n}=32)\end{array}$ & \\
\hline Ampicillin $(\leq 28)$ & 100 & 95.7 & 96.9 & 0.99 \\
\hline Clindamycin $(\leq 6)$ & 88.9 & 100 & 96.9 & 0.99 \\
\hline Penicillin $(\leq 28)$ & 100 & 91.3 & 93.8 & 0.68 \\
\hline Erythromycin $(\leq 13)$ & 88.9 & 95.7 & 93.8 & 0.98 \\
\hline Quinupristin/dalfopristin $(\leq 15)$ & 88.9 & 87.0 & 87.5 & 0.99 \\
\hline Streptomycin $(\leq 14)$ & 77.8 & 78.3 & 75.8 & 0.99 \\
\hline Teicoplanin $(\leq 10)$ & 77.8 & 73.9 & 75.0 & 0.99 \\
\hline Linezolid $(\leq 20)$ & 77.8 & 73.9 & 75.0 & 0.99 \\
\hline Vancomycin $(\leq 6)$ & 77.8 & 69.7 & 71.9 & 0.99 \\
\hline Kanamycin $(\leq 13)$ & 100 & 60.9 & 71.9 & 0.03 \\
\hline Tetracycline $(\leq 14)$ & 44.4 & 82.6 & 71.9 & 0.25 \\
\hline Gentamicin $(\leq 14)$ & 88.9 & 34.8 & 50.0 & 0.01 \\
\hline Chloramphenicol $(\leq 12)$ & 33.3 & 47.8 & 43.8 & 0.24 \\
\hline Ceftriaxone $(\leq 13)$ & 55.6 & 26.1 & 34.4 & 0.25 \\
\hline Ciprofloxacin $(\leq 15)$ & 22.2 & 13.0 & 15.6 & 0.87 \\
\hline Resistance to at least 1 antimicrobial class & 100 & 100 & 100 & \\
\hline Resistance to 3 or more antimicrobial classes & 88.9 & 100 & 96.9 & \\
\hline
\end{tabular}

${ }^{1}$ Comparison between fecal and milk isolates performed using Fisher's exact test.

$57.1 \%$ of the L. monocytogenes, E. coli $\mathrm{O} 157: \mathrm{H} 7$, and S. enterica isolates were resistant to 5 or more antimicrobial classes. In addition, $34.4,8.3$, and $17.9 \%$ of $L$. monocytogenes, E. coli O157:H7, and S. enterica were resistant to 9 or more antimicrobial classes (Supplemental Figure S1; https://doi.org/10.3168/jds.2019-16461).

\section{Antimicrobial Resistance Profiles}

We identified 22 unique antimicrobial resistance profiles in the L. monocytogenes isolates ( 6 by fecal isolates, 15 by milk isolates, and 1 shared by isolates from both sources). The $S$. enterica isolates exhibited 25 profiles (20 by fecal isolates, 4 by milk isolates, and 1 shared by isolates from both sources). The E. coli O157:H7 isolates exhibited 21 profiles (13 by RAMS and 8 by milk isolates). The profiles were diverse, and most were observed in a single isolate (Supplemental Tables S1, S2, and S3; https://doi.org/10.3168/jds.2019-16461).

\section{PFGE Typing of L. monocytogenes, S. enterica, and E. coli O157:H7}

The PFGE profiles of 2 L. monocytogenes isolates were highly dissimilar and had very different antimicrobial resistance phenotypes (Figure 1). The PFGE profiles of the $S$. enterica isolates were similar across farms, but had diverse antimicrobial resistance pheno-

Table 2. Antimicrobial resistance in Salmonella enterica isolates from dairy cattle in Jordan, 2016

\begin{tabular}{lcccc}
\hline & \multicolumn{3}{c}{ Resistance, $\%$} & \\
\cline { 2 - 4 } Antimicrobial (breakpoint, mm) & $\begin{array}{c}\text { Fecal isolates } \\
(\mathrm{n}=23)\end{array}$ & $\begin{array}{c}\text { Milk isolates } \\
(\mathrm{n}=5)\end{array}$ & $\begin{array}{c}\text { Total } \\
(\mathrm{n}=28)\end{array}$ & $P$-value \\
\hline Ampicillin $(\leq 13)$ & 82.6 & 100 & 85.7 & 0.99 \\
Cephalothin $(\leq 14)$ & 73.9 & 80.0 & 75.0 & 0.99 \\
Nalidixic acid $(\leq 13)$ & 69.6 & 80.0 & 71.4 & 0.99 \\
Kanamycin $(\leq 13)$ & 60.9 & 80.0 & 64.3 & 0.80 \\
Streptomycin $(\leq 11)$ & 52.2 & 80.0 & 55.2 & 0.53 \\
Amoxicillin-clavulanic acid $(\leq 13)$ & 56.5 & 60.0 & 57.1 & 0.99 \\
Tetracycline $(\leq 11)$ & 56.5 & 40.0 & 53.6 & 0.99 \\
Sulfamethoxazole-trimethoprim $(\leq 10)$ & 52.2 & 40.0 & 50.0 & 0.84 \\
Gentamicin $(\leq 12)$ & 30.4 & 60.0 & 35.7 & 0.46 \\
Chloramphenicol $(\leq 12)$ & 39.1 & 20.0 & 35.7 & 0.80 \\
Ciprofloxacin $(\leq 15)$ & 39.1 & 0 & 32.1 & 0.13 \\
Ceftriaxone $(\leq 19)$ & 17.4 & 20.0 & 17.9 & 0.99 \\
Resistance to at least 1 antimicrobial class & 100 & 100 & 100 & 82.1 \\
Resistance to 3 or more antimicrobial classes & 82.6 & 80.0 & & \\
\hline
\end{tabular}

${ }^{1}$ Comparison between fecal and milk isolates performed using Fisher's exact test. 
types. The PFGE profiles of E. coli $\mathrm{O} 157: \mathrm{H} 7$ isolates demonstrated homogeneity within and across farms. Two isolates from farm 27 were indistinguishable, 4 isolates from farm 5 were indistinguishable, and 1 isolate from farm 1 was indistinguishable from those in farm 5 . Isolates from farms 27, 5 , and 1 only differed by 1 band. Isolates with indistinguishable or highly similar PFGE profiles had diverse antimicrobial resistance profiles (Figure 1).

\section{DISCUSSION}

This study demonstrated a $21.3 \%$ prevalence of $L$. monocytogenes in BTM in Jordanian dairy cattle farms. Lower prevalence $(\leq 6 \%)$ in BTM samples has been reported in the United States (Sonnier et al., 2018), Spain (Vilar et al., 2007), New Zealand (Marshall et al., 2016), and Iran (Jamali et al., 2013). In dairy cattle feces, we found a $9.8 \%$ prevalence of L. monocytogenes in the present study, similar to the prevalence (9.3\%) detected in Spain (Vilar et al., 2007). The low prevalence of $L$. monocytogenes in dairy cattle farms in Jordan might be because the farms do not use silage as a feed component. Silage has been associated with the presence of L. monocytogenes in BTM (Sanaa et al., 1993; Vilar et al., 2007). The low prevalence might also be attributed to the small herd sizes on Jordanian dairy cattle farms; previous reports have demonstrated that larger herd size $(>500)$ increases the prevalence of L. monocytogenes (Antognoli et al., 2009). The higher prevalence of $L$. monocytogenes in BTM compared with that in feces could be attributed to the ability of this pathogen to form biofilms in milking parlor equipment (such as stainless steel pipelines) and farm environ- ments (Chmielewski and Frank, 2006; Latorre et al., 2010). Biofilm formation on milking parlor equipment could serve as a continuous source of BTM contamination as biofilm cells slough when milk passes through the pipelines (Chmielewski and Frank, 2006). In addition, the presence of $L$. monocytogenes in raw milk, even if pasteurized at a later stage, is a concern for dairy processing plants, because raw milk could be a source of plant contamination and consequently biofilm formation (Chmielewski and Frank, 2006; Latorre et al., 2010). Further studies in Jordan are recommended on biofilm presence on milking parlor equipment. The presence of $L$. monocytogenes in BTM and the ability of this bacteria to grow under refrigeration temperatures could pose a risk for human listeriosis.

This study showed a low prevalence $(3.3 \%)$ of S. enterica in BTM in Jordan dairies. Previously published studies did not detect $S$. enterica in in-line milk filters in dairy farms in Italy (Giacometti et al., 2012) or in New Zealand (Marshall et al., 2016), but higher prevalences were reported in the United States [4.7 and $10.1 \%$ in BTM and $31.9 \%$ in milk filters from the same farms (Ruzante et al., 2010) and 3.0\% in BTM and $18.0 \%$ in milk filters (Sonnier et al., 2018)]. In addition, we found a higher prevalence of $S$. enterica (16.4\%) in fecal samples in this study, but previous reports showed low prevalence in dairy cattle in Canada (3.3\%; Awosile et al., 2018) and Ethiopia (7.6\%; Eguale et al., 2016). These differences in $S$. enterica prevalence might be explained by variations in production practices, climate, and sampling scheme. In addition, differences in herd size could be an important factor, because herd size has been found to be a significant risk factor for $S$. enterica in BTM, with herd sizes of $>500$ at highest risk $(\mathrm{Ru}-$

Table 3. Antimicrobial resistance in Escherichia coli O157:H7 isolates from dairy cattle in Jordan, 2016

\begin{tabular}{|c|c|c|c|c|}
\hline \multirow[b]{2}{*}{ Antimicrobial (breakpoint, mm) } & \multicolumn{3}{|c|}{ Resistance, $\%$} & \multirow[b]{2}{*}{$P$-value ${ }^{2}$} \\
\hline & $\begin{array}{l}\mathrm{RAMS}^{1} \text { isolates } \\
\quad(\mathrm{n}=14)\end{array}$ & $\begin{array}{l}\text { Milk isolates } \\
\quad(\mathrm{n}=10)\end{array}$ & $\begin{array}{c}\text { Total } \\
(\mathrm{n}=24)\end{array}$ & \\
\hline Streptomycin $(\leq 11)$ & 92.9 & 90.0 & 91.2 & 0.99 \\
\hline Cefalothin $(\leq 14)$ & 85.7 & 100 & 91.7 & 0.99 \\
\hline Nalidixic acid $(\leq 13)$ & 71.4 & 90 & 79.2 & 0.57 \\
\hline Tetracycline $(\leq 11)$ & 78.6 & 80.0 & 79.2 & 0.99 \\
\hline Kanamycin $(\leq 13)$ & 35.7 & 90 & 58.3 & 0.02 \\
\hline Sulfamethoxazole-trimethoprim $(\leq 10)$ & 50 & 30 & 41.7 & 0.58 \\
\hline Gentamicin $(\leq 12)$ & 21.4 & 60.0 & 37.5 & 0.13 \\
\hline Chloramphenicol $(\leq 12)$ & 50.0 & 20.0 & 37.5 & 0.29 \\
\hline Ceftriaxone $(\leq 19)$ & 7.1 & 70 & 33.3 & 0.004 \\
\hline Ciprofloxacin $(\leq 15)$ & 21.4 & 0 & 12.5 & 0.18 \\
\hline Resistance to at least 1 antimicrobial class & 100 & 100 & 100 & \\
\hline
\end{tabular}

${ }^{1}$ RAMS $=$ rectoanal mucosal swab.

${ }^{2}$ Comparison between fecal and milk isolates performed using Fisher's exact test. 
zante et al., 2010; Giacometti et al., 2012). Shedding of $S$. enterica peaks during summer months (Pangloli et al., 2008), but the samples in this study were collected during the spring, which might not be a factor in detecting low prevalence, because no difference in $S$. enterica prevalence was found between spring and summer in a recent study from dairies in the United States (Ruzante et al., 2010). Hygienic milking practices are recommended to minimize milk contamination with fecal materials that carry foodborne pathogens. This can be challenging for farmers, because healthy animals can shed these pathogens in their feces.

Antimicrobials are used to treat L. monocytogenes infections in humans and animals, but resistance to antimicrobials has been reported in isolates from dairy products, animals, and human clinical cases (Sharma et al., 2017; Tahoun et al., 2017; Akrami-Mohajeri et al., 2018; Noll et al., 2018). Our results demonstrated that all L. monocytogenes isolates from Jordan cattle dairies (both fecal and milk isolates) were multidrugresistant. Similarly, high multidrug resistance (88\%) by L. monocytogenes isolates from dairy cattle farms and workers was recently reported in Egypt (Tahoun et al., 2017). The isolates from Jordan cattle dairies demonstrated high resistance $(95 \%)$ to ampicillin, penicillin, clindamycin, and erythromycin and high resistance toward quinupristin-dalfopristin (88\%) and streptomycin (80\%). In addition, more than $70 \%$ of the

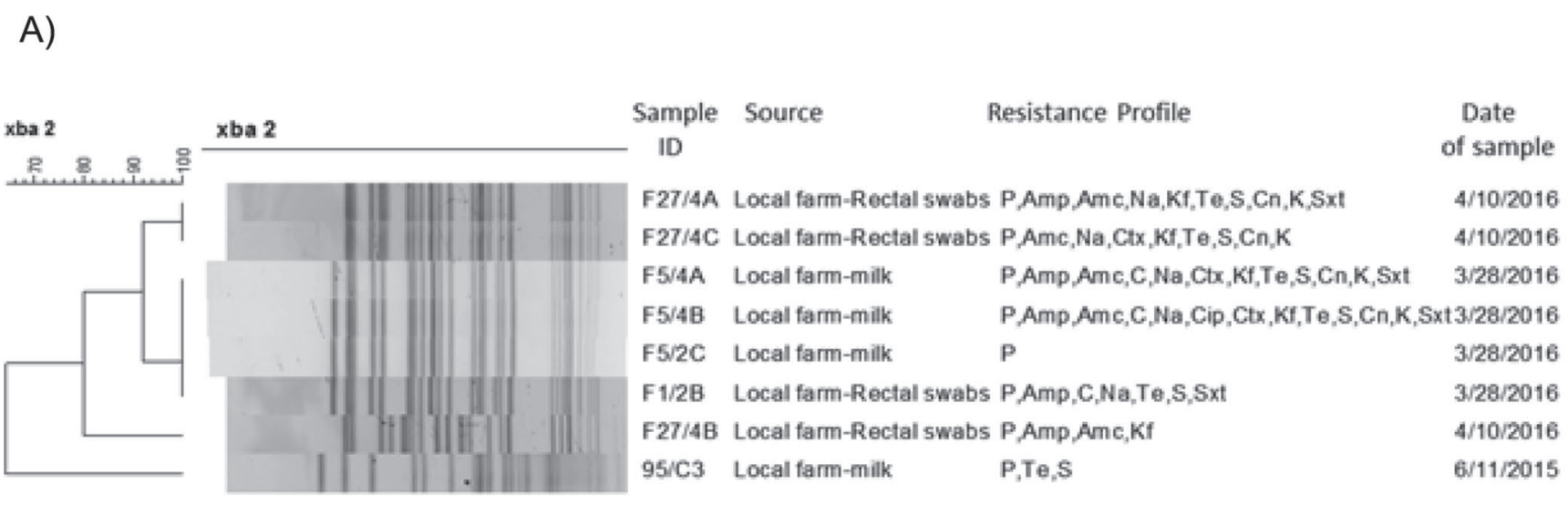

B)

\begin{tabular}{|c|c|c|c|c|c|c|}
\hline XEA & XBA & $\begin{array}{l}\text { Sample } \\
\text { ID }\end{array}$ & Source & Resistance profile & $\begin{array}{c}\text { Date } \\
\text { of sample }\end{array}$ & Serogroup \\
\hline & & $\mathrm{F} 21 \mathrm{~B}$ & Local farm-Feces & Amp Amx $\mathrm{C}_{2} \mathrm{Na}_{2} \mathrm{Ctx}, \mathrm{Te}, \mathrm{S}, \mathrm{Cn}, \mathrm{K}, \mathrm{Sxt}$ & $3 / 29 / 2016$ & $\mathrm{E} 1$ \\
\hline & & $\mathrm{F} 22 \mathrm{~A}$ & Local farm-Feces & Amp Amc,Kt & $3 / 29 / 2016$ & E1 \\
\hline & & $\mathrm{F} 36 \mathrm{~A}$ & Local farm-Feces & Amp Amc, $\mathrm{Na}_{z} \mathrm{KI}, \mathrm{Te}_{2} \mathrm{~S}, \mathrm{~K}, \mathrm{~S} x t$ & $4 / 24 / 2016$ & $\mathrm{E} 1$ \\
\hline & & F $36 \mathrm{C}$ & Local farm-Feces & Amp,Amc, C,Na, Cip,Kf,Te,Cn,K,Sxt & $4 / 24 / 2016$ & E1 \\
\hline & & F33/A & Local farm-Feces & Amp Amc,C,Na,Cip,Cb, Kf, Te,S,Sxt & $4 / 24 / 2016$ & E1 \\
\hline & & F $36 \mathrm{~B}$ & Local farm-Feces & Amp,Amc,Na,Cip,Te,S,K,Sxt & $4 / 24 / 2016$ & E1 \\
\hline & & $\mathrm{F} 1 \mathrm{C}$ & Local farm-Feces & $\mathrm{Na}$ & $3 / 27 / 2016$ & E1 \\
\hline & & F $38 B$ & Local farm-Feces & Amp, C,Na, Cip, Te, S, Cn, K, Sxt & $4 / 26 / 2016$ & $\mathrm{E} 1$ \\
\hline
\end{tabular}

C)

\begin{tabular}{|c|c|c|c|c|}
\hline Iisteria & $\begin{array}{c}\text { Sample } \\
\text { ID }\end{array}$ & Source & Resistance profile & $\begin{array}{l}\text { Date } \\
\text { of sample }\end{array}$ \\
\hline & $\mathrm{F} 32 \mathrm{C}$ & Local Farm-Feces & $\mathrm{P}, \mathrm{Ctx}, \mathrm{C}, \mathrm{E}, \mathrm{Da}, \mathrm{Te}, \mathrm{Cn}, \mathrm{K}, \mathrm{Od}, \mathrm{Tec}, \mathrm{Lzd}, \mathrm{Va}$ & $4 / 24 / 2016$ \\
\hline & F37A & Local Farm-Feces & PAmp,Cx, C,S,Cip,E,Da,Te, Cn, K, Od,Tec,Lzd,Va & $4 / 24 / 2016$ \\
\hline
\end{tabular}

Figure 1. (A) XbaI pulsed-field gel electrophoresis (PFGE) profiles of Escherichia coli O157:H7 from dairy farms in Jordan. (B) XbaI-PFGE profiles of Salmonella enterica from dairy farms in Jordan. (C) AscI-PFGE profiles of Listeria monocytogenes from dairy farms in Jordan. Amc, Amx = amoxicillin-clavulanic acid; Amp = ampicillin; $\mathrm{C}=$ chloramphenicol; $\mathrm{Cip}=$ ciprofloxacin $; \mathrm{Cn}=$ gentamicin; $\mathrm{Ctx}=$ cefotaxime; Da = clindamycin; $\mathrm{E}=$ erythromycin; $\mathrm{K}=$ kanamycin; $\mathrm{Kf}=$ cephalothin; Lzd = linezolid; $\mathrm{Na}=$ nalidixic acid; $\mathrm{P}=$ penicillin; $\mathrm{Qd}=$ quinupristin-dalfopristin; S = streptomycin; Sxt = sulfamethoxazole-trimethoprim; Te = tetracycline; Tec = teicoplanin; Va = vancomycin. 
isolates exhibited resistance to teicoplanin, linezolid, vancomycin, kanamycin, and tetracycline. Another study reported $100 \%$ resistance to penicillin, amoxicillin-clavulanic acid, tetracycline, and chloramphenicol in isolates from dairy products in Yazd, Iran (AkramiMohajeri et al., 2018). High resistance (>65\%) toward tetracycline, doxycycline, clindamycin, rifampicin, gentamicin, and daptomycin by $L$. monocytogenes from raw milk, milking equipment, and workers was found in Egypt (Tahoun et al., 2017). Moreover, high resistance $(>80 \%)$ was exhibited by isolates from raw milk in India toward ampicillin, penicillin G, amoxicillin-clavulanic acid, ceftriaxone, and nalidixic acid (Sharma et al., 2017). The high multidrug resistance and high resistance percentages to the antimicrobial drugs of choice for L. monocytogenes treatment (such as $\beta$-lactams and aminoglycosides) found in this study and in the above-mentioned countries is alarming, because this resistance can increase fatality rates (Thønnings et al., 2016). Sulfamethoxazole-trimethoprim has been proposed as an alternative for listeriosis treatment (Fernández Guerrero et al., 2012), but we did not test the susceptibility or resistance of the L. monocytogenes isolates to this antimicrobial in the present study.

A very high percentage $(80 \%)$ of the $S$. enterica isolates from Jordan cattle dairies exhibited multidrug resistance. In comparison, much lower (10\%) multidrug resistance was reported in other locations such as Texas, several other states in the United States, and Thailand (Chuanchuen et al., 2010; Rodriguez-Rivera et al., 2014; Sonnier et al., 2018). One study in Ethiopia reported high $(70 \%)$ multidrug resistance in fecal isolates (Eguale et al., 2016). In addition, all S. enterica were resistant to at least 1 antimicrobial in the present study; however, lower resistance toward at least 1 antibiotic was exhibited by isolates from dairy farms in the United States (14 and 20\%; Rodriguez-Rivera et al., 2016; Sonnier et al., 2018), but high percentages were reported in Thailand (68\%; Chuanchuen et al., 2010). Of the isolates in this study, 30 and $16.7 \%$ exhibited resistance to ciprofloxacin and ceftriaxone, respectively. This resistance is concerning, because ciprofloxacin and ceftriaxone are used to treat invasive salmonellosis in adults and children (Chiappini et al., 2002; Chen et al., 2013). A recent study in the United States showed that $S$. enterica resistance to ceftriaxone in human isolates correlated with resistance from cattle and their products (Iwamoto et al., 2017). Resistance to ceftriaxone might be due to the widespread use of ceftiofur in Jordan cattle dairies for metritis treatment, because previous reports have highlighted that using ceftiofur can co-select for ceftriaxone resistance (Teshager et al., 2000; Obaidat et al., 2018).
Limited data are available on the antimicrobial resistance to E. coli O157:H7. In the present study, a high percentage (92\%) of the E. coli $\mathrm{O} 157: \mathrm{H} 7$ isolates from Jordan cattle farms exhibited multidrug resistance . A previous study reported that all E. coli $\mathrm{O} 157: \mathrm{H} 7$ isolates from sheep were resistant toward sulphonamides and chloramphenicol, and $62.5 \%$ were also resistant toward ampicillin and streptomycin (Novotna et al., 2005).

The present study highlighted differences in antibiotic resistance among fecal and milk isolates. These differences were not significant in the majority of cases. However, fecal L. monocytogenes isolates exhibited significantly higher resistance to kanamycin and gentamicin than milk isolates. In contrast, milk E. coli $\mathrm{O} 157: \mathrm{H} 7$ isolates exhibited significantly higher resistance toward kanamycin and ceftriaxone than RAMS isolates. The lower resistance demonstrated toward ceftriaxone in RAMS isolates could be attributed to the ability of the microflora in the cattle large intestine to degrade this antimicrobial and ceftiofur by multiple $\beta$-lactamases (Wagner et al., 2011). Higher prevalences of multidrugresistant $S$. enterica have been reported in dairy cow feces compared with dairy farm environmental samples in Texas (Rodriguez-Rivera et al., 2016). Higher resistance in fecal isolates to $S$. enterica compared with milk isolates for gentamicin, kanamycin, nitrofurantoin, and tetracycline have also been reported in Ethiopia (Addis et al., 2011), but these differences were not significant. In most cases, the differences in the present study and the Ethiopian study could be attributed to the low number of isolates. Therefore, we recommend that future studies explore differences in antibiotic resistance among fecal and milk isolates using a larger number of isolates.

Both E. coli O157:H7 and S. enterica isolates in the present study shared a high similarity of individual isolates within species and across farms. This similarity can be explained by the persistence of these genotypes in the farm environment. The persistence of L. monocytogenes genotypes has been reported in Finnish dairy cattle farms (Castro et al., 2018). However, the PFGE profile similarity was not concordant with the antimicrobial resistance profiles, suggesting that certain resistance was likely to be associated with plasmids that were not shared among strains, and these plasmid differences could not be detected using PFGE.

\section{CONCLUSIONS}

Antimicrobial resistance is a cross-cutting threat to global health. Resistance identified and developed in the dairy systems of one country do not respect international borders, and have the potential to affect 
dairy systems in other countries. A robust evidence base that accurately describes the global burden of antimicrobial resistance will be essential for mitigating this challenge and informing global interventions. We detected a substantial prevalence of pathogens in dairy cattle in Jordan. Moreover, the prevalence and extent of the antimicrobial resistance exhibited by the 3 studied pathogens was high. This indicates that apparently healthy cattle in Jordan cattle dairies could be a source of multidrug-resistant foodborne pathogens affecting critically important antibiotics. These pathogens can be transmitted by consuming raw milk products, by direct contact with ruminants, and through environmental contamination. The establishment of an integrated surveillance system for human and animal foodborne pathogens across the food chain is recommended. In addition, educational programs about the adverse health consequences of raw milk product consumption is essential in Jordan. Further recommendations include educational programs for farmers, enacting regulations to restrict access to clinically important antimicrobials, developing guidelines for veterinarians, and research into the potential drivers and mitigation strategies needed to promote antimicrobial stewardship in dairy production in Jordan.

\section{ACKNOWLEDGMENTS}

The authors thank Margaret A. Davis from Paul Allen School for Global Animal Health at Washington State University for her technical advice and support. The authors also acknowledge the assistance of Alaa E. Bani Salman and Amany K. Rashaideh from Jordan University of Science and Technology. This work was supported by the Scientific Research Support Fund of the Jordanian Ministry of Higher Education and Scientific Research (Project \# Agri 49). The authors declare no conflict of interest.

\section{REFERENCES}

Addis, Z., N. Kebede, Z. Worku, H. Gezahegn, A. Yirsaw, and T. Kassa. 2011. Prevalence and antimicrobial resistance of Salmonella isolated from lactating cows and in contact humans in dairy farms of Addis Ababa: A cross sectional study. BMC Infect. Dis. 11:222.

Adhikari, B., T. E. Besser, J. M. Gay, L. K. Fox, M. A. Davis, R. N. Cobbold, A. C. Berge, R. McClanahan, and D. D. Hancock. 2009. Introduction of new multidrug-resistant Salmonella enterica strains into commercial dairy herds. J. Dairy Sci. 92:4218-4228.

Afema, J. A., A. E. Mather, and W. M. Sischo. 2015. Antimicrobial resistance profiles and diversity in Salmonella from humans and cattle, 2004-2011. Zoonoses Public Health 62:506-517.

Akrami-Mohajeri, F., Z. Derakhshan, M. Ferrante, N. Hamidiyan, M. Soleymani, G. O. Conti, and R. D. Tafti. 2018. The prevalence and antimicrobial resistance of Listeria spp in raw milk and traditional dairy products delivered in Yazd, central Iran (2016). Food Chem. Toxicol. 114:141-144.
Antognoli, M. C., J. E. Lombard, B. A. Wagner, B. J. McCluskey, J. S. Van Kessel, and J. S. Karns. 2009. Risk factors associated with the presence of viable Listeria monocytogenes in bulk tank milk from US dairies. Zoonoses Public Health 56:77-83.

Arthur, T. M., D. M. Brichta-Harhay, J. M. Bosilevac, N. Kalchayanand, S. D. Shackelford, T. L. Wheeler, and M. Koohmaraie. 2010. Super shedding of Escherichia coli O157:H7 by cattle and the impact on beef carcass contamination. Meat Sci. 86:32-37.

Awosile, B., J. McClure, J. Sanchez, J. C. Rodriguez-Lecompte, G. Keefe, and L. C. Heider. 2018. Salmonella enterica and extendedspectrum cephalosporin-resistant Escherichia coli recovered from Holstein dairy calves from 8 farms in New Brunswick, Canada. J. Dairy Sci. 101:3271-3284.

Berge, A. C., S. C. Champagne, R. M. Finger, and W. M. Sischo. 2007. The use of bulk tank milk samples to monitor trends in antimicrobial resistance on dairy farms. Foodborne Pathog. Dis. 4:397-407.

Castro, H., A. Jaakkonen, M. Hakkinen, H. Korkeala, and M. Lindstrom. 2018. Occurrence, persistence, and contamination routes of Listeria monocytogenes genotypes on three Finnish dairy cattle farms: A longitudinal study. Appl. Environ. Microbiol. 84;e0200017.

CDC (Centers for Disease Control and Prevention). 2008. Outbreak of Listeria monocytogenes infections associated with pasteurized milk from a local dairy-Massachusetts, 2007. MMWR Morb. Mortal. Wkly. Rep. 57:1097-1100.

Chen, H. M., Y. Wang, L. H. Su, and C. H. Chiu. 2013. Nontyphoid salmonella infection: Microbiology, clinical features, and antimicrobial therapy. Pediatr. Neonatol. 54:147-152.

Chiappini, E., L. Galli, P. Pecile, A. Vierucci, and M. de Martino. 2002. Results of a 5-year prospective surveillance study of antibiotic resistance among Salmonella enterica isolates and ceftriaxone therapy among children hospitalized for acute diarrhea. Clin. Ther. 24:1585-1594.

Chmielewski, R. A. N., and J. Frank. 2006. Biofilm formation and control in food processing facilities. Compr. Rev. Food Sci. Food Saf. 2:22-32.

Chuanchuen, R., K. Ajariyakhajorn, C. Koowatananukul, W. Wannaprasat, S. Khemtong, and S. Samngamnim. 2010. Antimicrobial resistance and virulence genes in Salmonella enterica isolates from dairy cows. Foodborne Pathog. Dis. 7:63-69.

CLSI (Clinical and Laboratory Standards Institute). 2014. Performance Standards for Antimicrobial Susceptibility Testing; Twenty-Fourth Informational Supplement, CLSI document M100-S24. Clinical and Laboratory Standards Institute, Wayne, PA.

Currie, A., E. Galanis, P. A. Chacon, R. Murray, L. Wilcott, P. Kirkby, L. Honish, K. Franklin, J. Farber, R. Parker, S. Shyng, D. Sharma, L. Tschetter, L. Hoang, L. Chui, A. Pacagnella, J. Wong, J. Pritchard, A. Kerr, M. Taylor, V. Mah, and J. Flint. 2018. Outbreak of Escherichia coli O157:H7 infections linked to aged raw milk gouda cheese, Canada, 2013. J. Food Prot. 81:325-331.

D'Agostino, M., M. Wagner, J. A. Vazquez-Boland, T. Kuchta, R. Karpiskova, J. Hoorfar, S. Novella, M. Scortti, J. Ellison, A. Murray, I. Fernandes, M. Kuhn, J. Pazlarova, A. Heuvelink, and N. A. Cook. 2004. validated PCR-based method to detect Listeria monocytogenes using raw milk as a food model-towards an international standard. J. Food Prot. 67:1646-1655.

Davis, M. A., D. H. Rice, H. Sheng, D. D. Hancock, T. E. Besser, R. Cobbold, and C. J. Hovde. 2006. Comparison of cultures from rectoanal-junction mucosal swabs and feces for detection of Escherichia coli O157 in dairy heifers. Appl. Environ. Microbiol. 72:3766-3770.

Dean, A. G., M. Sullivan, and M. M. Soe. 2015. OpenEpi: Open source epidemiologic statistics for public health, version 3.01. Accessed Jun. 21, 2019. www.OpenEpi.com.

Eguale, T., D. Asrat, H. Alemayehu, I. Nana, W. A. Gebreyes, J. S. Gunn, and E. Engidawork. 2018. Phenotypic and genotypic characterization of temporally related nontyphoidal Salmonella strains isolated from humans and food animals in central Ethiopia. Zoonoses Public Health 65:766-776.

Eguale, T., E. Engidawork, W. A. Gebreyes, D. Asrat, H. Alemayehu, G. Medhin, R. P. Johnson, and J. S. Gunn. 2016. Fecal prevalence, 
serotype distribution and antimicrobial resistance of Salmonellae in dairy cattle in central Ethiopia. BMC Microbiol. 16:20.

Fernández Guerrero, M. L., R. Torres, B. Mancebo, J. J. GonzálezLópez, M. Górgolas, J. J. Jusdado, and R. F. Roblas. 2012. Antimicrobial treatment of invasive non-perinatal human listeriosis and the impact of the underlying disease on prognosis. Clin. Microbiol. Infect. 18:690-695.

Giacometti, F., A. Serraino, G. Finazzi, P. Daminelli, M. N. Losio, P. Bonilauri, N. Arrigoni, A. Garigliani, R. Mattioli, S. Alonso, S. Piva, D. Florio, R. Riu, and R. G. Zanoni. 2012. Foodborne pathogens in in-line milk filters and associated on-farm risk factors in dairy farms authorized to produce and sell raw milk in northern Italy. J. Food Prot. 75:1263-1269.

Greenquist, M. A., J. S. Drouillard, J. M. Sargeant, B. E. Depenbusch, X. Shi, K. F. Lechtenberg, and T. G. Nagaraja. 2005. Comparison of rectoanal mucosal swab cultures and fecal cultures for determining prevalence of Escherichia coli O157:H7 in feedlot cattle. Appl. Environ. Microbiol. 71:6431-6433.

Heiman, K. E., R. K. Mody, S. D. Johnson, P. M. Griffin, and L. H. Gould. 2015. Escherichia coli O157 outbreaks in the United States, 2003-2012. Emerg. Infect. Dis. 21:1293-1301.

Helke, K. L., M. A. McCrackin, A. M. Galloway, A. Z. Poole, C. D. Salgado, and B. P. Marriott. 2017. Effects of antimicrobial use in agricultural animals on drug-resistant foodborne salmonellosis in humans: A systematic literature review. Crit. Rev. Food Sci. Nutr. $57: 472-488$.

Hilali, M., E. El-Mayda, and B. Rischkowsky. 2011. Characteristics and utilization of sheep and goat milk in the Middle East. Small Rumin. Res. 101:92-101.

Holmes, A. H., L. S. Moore, A. Sundsfjord, M. Steinbakk, S. Regmi, A. Karkey, P. J. Guerin, and L. J. Piddock. 2016. Understanding the mechanisms and drivers of antimicrobial resistance. Lancet 387:176-187.

Iwamoto, M., J. Reynolds, B. E. Karp, H. Tate, P. J. Fedorka-Cray, J. R. Plumblee, R. M. Hoekstra, J. M. Whichard, and B. E. Mahon. 2017. Ceftriaxone-resistant nontyphoidal Salmonella from humans, retail meats, and food animals in the United States, 1996-2013. Foodborne Pathog. Dis. 14:74-83.

Jackson, K. A., M. Biggerstaff, M. Tobin-D'Angelo, D. Sweat, R. Klos, J. Nosari, O. Garrison, E. Boothe, L. Saathoff-Huber, L. Hainstock, and R. P. Fagan. 2011. Multistate outbreak of Listeria monocytogenes associated with Mexican-style cheese made from pasteurized milk among pregnant, Hispanic women. J. Food Prot. 74:949-953.

Jamali, H., B. Radmehr, and K. L. Thong. 2013. Prevalence, characterisation, and antimicrobial resistance of Listeria species and Listeria monocytogenes isolates from raw milk in farm bulk tanks. Food Control 34:121-125.

Jensen, A. K., J. T. Björkman, S. Ethelberg, K. Kiil, M. Kemp, and E. M. Nielsen. 2016. Molecular typing and epidemiology of human listeriosis cases, Denmark, 2002-2012. Emerg. Infect. Dis. 22:625-633.

Langer, A. J., T. Ayers, J. Grass, M. Lynch, F. J. Angulo, and B. E. Mahon. 2012. Nonpasteurized dairy products, disease outbreaks, and State laws-United States, 1993-2006. Emerg. Infect. Dis. 18:385-391.

Latorre, A. A., J. S. Van Kessel, J. S. Karns, M. J. Zurakowski, A. K. Pradhan, K. J. Boor, B. M. Jayarao, B. A. Houser, C. S. Daugherty, and Y. H. Schukken. 2010. Biofilm in milking equipment on a dairy farm as a potential source of bulk tank milk contamination with Listeria monocytogenes. J. Dairy Sci. 93:2792-2802.

Magiorakos, A. P., A. Srinivasan, R. B. Carey, Y. Carmeli, M. E. Falagas, C. G. Giske, S. Harbarth, J. F. Hindler, G. Kahlmeter, B. Olsson-Liljequist, D. L. Paterson, L. B. Rice, J. Stelling, M. J. Struelens, A. Vatopoulos, J. T. Weber, and D. L. Monnet. 2012. Multidrug-resistant, extensively drug-resistant and pandrug-resistant bacteria: an international expert proposal for interim standard definitions for acquired resistance. Clin. Microbiol. Infect. 18:268-281.
Marshall, J. C., T. K. Soboleva, P. Jamieson, and N. P. French. 2016. Estimating bacterial pathogen levels in New Zealand bulk tank milk. J. Food Prot. 79:771-780.

Mohammed, H. O., K. Stipetic, P. L. McDonough, R. N. Gonzalez, D. V. Nydam, and E. R. Atwill. 2009. Identification of potential on-farm sources of Listeria monocytogenes in herds of dairy cattle. Am. J. Vet. Res. 70:383-388.

Mungai, E. A., C. B. Behravesh, and L. H. Gould. 2015. Increased outbreaks associated with nonpasteurized milk, United States, 2007-2012. Emerg. Infect. Dis. 21:119-122.

Noll, M., S. Kleta, and S. Al Dahouk. 2018. Antibiotic susceptibility of 259 Listeria monocytogenes strains isolated from food, food-processing plants and human samples in Germany. J. Infect. Public Health 11:572-577.

Novotna, R., P. Alexa, J. Hamrik, A. Madanat, J. Smola, and A. Cizek. 2005. Isolation and characterization Shiga toxin-producing Escherichia coli from sheep and goats in Jordan with evidence of multiresistant serotype O157:H7. Vet. Med. Czech 50:111-118.

Obaidat, M. M. 2019. Seroprevalence and risk factors for Campylobacter jejuni seropositivity in Jordan. Infect. Dis. (Lond.) 51:140146 .

Obaidat, M. M., and A. E. Bani Salman. 2017. Antimicrobial resistance percentages of Salmonella and Shigella in seafood imported to Jordan: higher percentages and more diverse profiles in Shigella. J. Food Prot. 80:414-419

Obaidat, M. M., A. E. Bani Salman, M. A. Davis, and A. A. Roess. 2018. Major diseases, extensive misuse, and high antimicrobial resistance of Escherichia coli in large- and small-scale dairy cattle farms in Jordan. J. Dairy Sci. 101:2324-2334.

Pangloli, P., Y. Dje, O. Ahmed, C. A. Doane, S. P. Oliver, and F. A. Draughon. 2008. Seasonal incidence and molecular characterization of Salmonella from dairy cows, calves, and farm environment. Foodborne Pathog. Dis. 5:87-96.

Pennington, H. 2010. Escherichia coli O157. Lancet 376:1428-1435.

Pornsukarom, S., A. H. M. van Vliet, and S. Thakur. 2018. Whole genome sequencing analysis of multiple Salmonella serovars provides insights into phylogenetic relatedness, antimicrobial resistance, and virulence markers across humans, food animals and agriculture environmental sources. BMC Genomics 19:801.

Pouillot, R., K. C. Klontz, Y. Chen, L. S. Burall, D. Macarisin, M. Doyle, K. M. Bally, E. Strain, A. R. Datta, T. S. Hammack, and J. M. Van Doren. 2016. Infectious dose of Listeria monocytogenes in outbreak linked to ice cream, United States, 2015. Emerg. Infect. Dis. 22:2113-2119.

Rice, D. H., H. Q. Sheng, S. A. Wynia, and C. J. Hovde. 2003. Rectoanal mucosal swab culture is more sensitive than fecal culture and distinguishes Escherichia coli O157:H7-colonized cattle and those transiently shedding the same organism. J. Clin. Microbiol. 41:4924-4929.

Rodriguez-Rivera, L. D., K. J. Cummings, G. H. Loneragan, S. C. Rankin, D. L. Hanson, W. M. Leone, and T. S. Edrington. 2016. Salmonella prevalence and antimicrobial susceptibility among dairy farm environmental samples collected in Texas. Foodborne Pathog. Dis. 13:205-211.

Rodriguez-Rivera, L. D., E. M. Wright, J. D. Siler, M. Elton, K. J. Cummings, L. D. Warnick, and M. Wiedmann. 2014. Subtype analysis of Salmonella isolated from subclinically infected dairy cattle and dairy farm environments reveals the presence of both human- and bovine-associated subtypes. Vet. Microbiol. 170:307316

Ruzante, J. M., J. E. Lombard, B. Wagner, C. P. Fossler, J. S. Karns, J. A. Van Kessel, and I. A. Gardner. 2010. Factors associated with Salmonella presence in environmental samples and bulk tank milk from US dairies. Zoonoses Public Health 57:e217-e225.

Sanaa, M., B. Poutrel, J. L. Menard, and F. Serieys. 1993. Risk factors associated with contamination of raw milk by Listeria monocytogenes in dairy farms. J. Dairy Sci. 76:2891-2898.

Scallan, E., R. M. Hoekstra, F. J. Angulo, R. V. Tauxe, M. A. Widdowson, S. L. Roy, J. L. Jones, and P. M. Griffin. 2011. Foodborne 
illness acquired in the United States - major pathogens. Emerg. Infect. Dis. 17:7-15.

Schoder, D., D. Melzner, A. Schmalwieser, A. Zangana, P. Winter, and M. Wagner. 2011. Important vectors for Listeria monocytogenes transmission at farm dairies manufacturing fresh sheep and goat cheese from raw milk. J. Food Prot. 74:919-924.

Sharma, S., V. Sharma, D. K. Dahiya, A. Khan, M. Mathur, and A. Sharma. 2017. Prevalence, virulence potential, and antibiotic susceptibility profile of Listeria monocytogenes isolated from bovine raw milk samples obtained from Rajasthan, India. Foodborne Pathog. Dis. 14:132-140.

Sheng, H., Y. N. Nguyen, C. J. Hovde, and V. Sperandio. 2013. SdiA aids enterohemorrhagic Escherichia coli carriage by cattle fed a forage or grain diet. Infect. Immun. 81:3472-3478.

Sheng, H., S. Shringi, K. N. Baker, S. A. Minnich, C. J. Hovde, and T. E. Besser. 2015. Standardized Escherichia coli O157:H7 exposure studies in cattle provide evidence that bovine factors do not drive increased summertime colonization. Appl. Environ. Microbiol. 82:964-971.

Sonnier, J. L., J. S. Karns, J. E. Lombard, C. A. Kopral, B. J. Haley, S. W. Kim, and J. A. S. Van Kessel. 2018. Prevalence of Salmonella enterica, Listeria monocytogenes, and pathogenic Escherichia coli in bulk tank milk and milk filters from US dairy operations in the National Animal Health Monitoring System Dairy 2014 study. J. Dairy Sci. 101:1943-1956.

Tahoun, A. B. M. B., R. M. M. Abou Elez, E. N. Abdelfatah, I. Elsohaby, A. A. El-Gedawy, and A. M. Elmoslemany. 2017. Listeria monocytogenes in raw milk, milking equipment and dairy workers: Molecular characterization and antimicrobial resistance patterns. J. Glob. Antimicrob. Resist. 10:264-270.

Teshager, T., I. A. Herrero, M. C. Porrero, J. Garde, M. A. Moreno, and L. Dominguez. 2000. Surveillance of antimicrobial resistance in Escherichia coli strains isolated from pigs at Spanish slaughterhouses. Int. J. Antimicrob. Agents 15:137-142.

Thønnings, S., J. D. Knudsen, H. C. Schønheyder, M. Søgaard, M. Arpi, K. O. Gradel, C. Østergaard, C. Østergaard, M. Arpi, K. O. Gradel, U. S. Jensen, S. Thønnings, J. D. Knudsen, K. Koch, M. Pinholt, J. Smit, H. C. Schønheyder, and M. Søgaard. 2016. Antibiotic treatment and mortality in patients with Listeria monocytogenes meningitis or bacteraemia. Clin. Microbiol. Infect. 22:725-730.

Van Kessel, J. A., J. S. Karns, J. E. Lombard, and C. A. Kopral. 2011. Prevalence of Salmonella enterica, Listeria monocytogenes, and Escherichia coli virulence factors in bulk tank milk and in-line filters from U.S. dairies. J. Food Prot. 74:759-768.

Van Kessel, J. S., J. Sonnier, S. Zhao, and J. S. Karns. 2013. Antimicrobial resistance of Salmonella enterica isolates from bulk tank milk and milk filters in the United States. J. Food Prot. 76:18-25.

Vilar, M. J., E. Yus, M. L. Sanjuan, F. J. Dieguez, and J. L. Rodriguez-Otero. 2007. Prevalence of and risk factors for Listeria species on dairy farms. J. Dairy Sci. 90:5083-5088.

Wagner, R. D., S. J. Johnson, C. E. Cerniglia, and B. D. Erickson. 2011. Bovine intestinal bacteria inactivate and degrade ceftiofur and ceftriaxone with multiple $\beta$-lactamases. Antimicrob. Agents Chemother. 55:4990-4998.

WHO (World Health Organization). 2015. WHO Estimates of the global burden of foodborne diseases: foodborne disease burden epidemiology reference group 2007-2015. Accessed Dec. 7, 2018. http: //apps.who.int/iris/bitstream/handle/10665/199350/?sequence= 1.

WHO (World Health Organization). 2017. Global Antimicrobial Resistance Surveillance System (GLASS) report: Early Implementation 2016-2017. WHO, Geneva, Switzerland. 